Enferm Bras 2020;19(4Supl):S3-S5

https://doi.org/10.33233/eb.v19i4.4378

\title{
OPINIÃO \\ COVID-19 no idoso: qual seria o momento ideal para iniciar atividade física no âmbito hospitalar?
}

Marco Antônio Orsini Neves ${ }^{1}$, Mauricio de Sant Anna $\mathrm{Jr}^{2}$, Marco Antônio Alves Azizi ${ }^{3}$, Renata Rodrigues Teixeira Castro ${ }^{4}$, Janie Kelly Fernandes do Nascimento ${ }^{5}$, Nicolle dos Santos Moraes Nunes ${ }^{5}$, Jacqueline Stephanie Fernandes do Nascimento ${ }^{5}$

${ }^{1}$ Médico, Professor Titular da Universidade Iguaçu - UNIG-RJ, Nova Iguaçu/RJ, Brasil, Professor Titular da Universidade de Vassouras - USS-RJ, Vassouras/RJ, Brasil, ${ }^{2}$ Fisioterapeuta, Professor Adjunto do Instituto Federal do Rio de Janeiro (IFRJ), ${ }^{3}$ Médico, Professor Titular da Universidade Iguaçu UNIG-RJ, Nova Iguaçu/RJ, ${ }^{4}$ Médica, Professor Titular da Universidade Iguaçu - UNIG-RJ, Nova Iguaçu/RJ, ${ }^{5}$ Graduanda da escola de medicina da Universidade Iguaçu, Universidade Iguaçu- UNIG-RJ, Nova Iguaçu/RJ, Brasil

Recebido 15 de julho de 2020; aceito 15 de agosto de 2020.

Correspondência: Mauricio de Sant Anna Jr, Rua Prof. Carlos Wenceslau, 343 Realengo 21715-000 Rio de Janeiro RJ.

Marco Antônio Orsini Neves: orsinimarco@hotmail.com

Mauricio de Sant Anna Jr: mauricio.junior@ifrj.edu.br

Marco Antônio Alves Azizi: marcoazizimed@gmail.com

Renata Rodrigues Teixeira de Castro: castrorrt@gmail.com

Janie Kelly Fernandes do Nascimento: janiekelly@hotmail.com

Nicolle dos Santos Moraes Nunes: nicolle.nunes_@hotmail.com

Jacqueline Stephanie Fernandes do Nascimento: jac.fn@hotmail.com

Adatar do ínicio da pandemia da COVID-19, muito se dedicou a conter a transmissão viral. No entanto, já ocorre uma tendência também em se preocupar com os pacientes sobreviventes à infecção da SARS-CoV-2. Isso se dá pelo fato de que a moléstia desenvolve um forte embate na saúde física, mental e psicossocial, independentemente da gravidade pela qual o paciente foi submetido, podendo manifestar, cronicamente, fraqueza muscular, dor, disfunção pulmonar, ansiedade, depressão e redução da qualidade de vida. Para resolução dessa temática, a reabilitação pulmonar se mostrou como o meio terapêutico mais benéfico ao qual tais pacientes possam ser submetidos [1,2].

As manifestações clínicas da COVID-19 são muito variáveis, porém, é sabido que os infectados possuem uma grande probabilidade de desenvolver distúrbios respiratórios, como a síndrome do desconforto respiratório agudo e a hipoxemia, que podem desencadear a falência múltipla de órgãos. Dependendo do curso clínico da doença, deve-se lançar mão de oxigenioterapia, ventilação mecânica não invasiva, cateter de alto fluxo, quando necessário em casos mais graves e refratários, intubação orotraqueal e internação em unidade de terapia intensiva [3].

Pacientes portadores de síndrome coronária, insuficiência cardíaca e que possuem revascularização miocárdica prévia são candidatos à reabilitação cardíaca. Para tanto, alguns critérios devem ser seguidos, conforme a Associação de Prevenção e Reabilitação Cardiovascular impõem: mudança no estilo de vida, práticas desportivas, terapia para transtornos mentais e psicológicos, visando melhorias na qualidade de vida, redução da morbimortalidade e possibilidade de retorno a atividades laborais [4].

Ao que se concerne às alterações musculo-esqueléticas, sabe-se que os pacientes que necessitaram de suporte ventilatório em UTI obtiveram maior chance de desenvolver fraqueza, hipotrofias e polineuropatia associada a doenças críticas. Dentre esses, a mais temida é osteonecrose induzida por altas doses de corticosteroides. Alguns estudos comprovaram que pacientes submetidos a internação em unidade de terapia intensiva por tempo prolongado, 
podem manifestar um conjunto de distúrbios físicos, psicológicos e cognitivos, caracterizados pela Síndrome pós-cuidados intensivos (PICS) [4,5].

Dentro desse contexto, a reabilitação pulmonar é amplamente difundida, sendo entendida como uma ingerência multidisciplinar que objetiva um tratamento individualizado, atendendo às necessidades impostas pelo paciente, a fim de se obter êxito na condição física, psíquica e social dos pacientes que sofreram afecções respiratórias. Os objetivos propostos pelas diretrizes que advogam a reabilitação pulmonar visam, a curto prazo, aliviar os sintomas de dispneia, como também a ansiedade e a depressão. Já nos objetivos a longo prazo, o foco se dirige a preservação da função pulmonar, otimização da qualidade de vida e reintrodução do paciente em seu meio de convívio $[4,6]$.

Pesquisas com nível de alta propriedade descreveram que a reabilitação pulmonar é favorável para pacientes que se encontram hospitalizados, ambulatoriais e em domicílio. Ainda assim, é válido ressaltar que a transição entre a fase de hospitalar e a transferência para um serviço voltado para a reabilitação deve ser paulatina. Além disso, não se deve iniciar precocemente a reabilitação, na fase aguda, que deve ser bem conduzida quanto a sua intensidade, frequência e duração para que possa ser bem tolerada $[7,8]$.

Segundo recomendações impostas pela Itália e China, não se deve iniciar precocemente a conduta de reabilitação respiratória, pois, paradoxalmente, é possível que haja deterioração respiratória e dispersação viral. Logo, na fase aguda, é importante que se tenha atenção quanto a utilização de técnicas que promovam aerossol em especial higiene brônquica, além de se evidar o aumento do consumo de oxigênio $[9,10]$.

Assim que se atingir a fase pós-aguda, a reabilitação respiratória deve ser instituída, com monitorização adequada a fim de se evitar sobrecargas ao sistema respiratório ou desencadear uma angústia respiratória aguda. A partir do começo da conduta de reabilitação, estes pacientes devem ser monitorados após a fase aguda da moléstia, mesmo quando se trata de pacientes mais jovens, é possível que desenvolvam alguns distúrbios respiratórios, como a SARS e a hipoxemia, gerando repercussões hemodinâmica com curso clínico bastante duvidoso $[9,11,12]$.

Para a realização do procedimento seguro, deve-se levar em consideração a condição física do indivíduo, como doenças de base, sinais vitais e contraindicações, associado a questões psíquicas e nutricionais. Dessa maneira, ao introduzir a reabilitação, também deve ser levado em conta a proteção que este procedimento confere ao paciente, devendo ser imediatamente interrompida se o paciente apresentar sinais de descompensação, sudorese, angina ou dispneia. Se a condição clínica do paciente for leve a moderada, o procedimento de reabilitação deve ser prontamente administrado, porém, em casos graves, este só pode ser implementado quando o paciente se encontrar estável hemodinamicamente [7,13].

A reabilitação pulmonar em pacientes que sofreram com SARS, segundo evidências, é extremamente importante. Isso porque foi comprovado que muitos doentes que obtiveram alta médica após tratamento para COVID-19 ainda podem manifestar sintomas pulmonares, como distúrbios restritivos, e dispneia ao realizarem esforços, afetando negativamente suas atividades laborais, além da qualidade de vida. Admite-se que esta problemática advenha de um longo repouso, efeitos colaterais do tratamento medicamento e possível resíduo da moléstia, como alveolite persistente, fibrose, atelectasia e níveis variáveis de disfunção, gerando uma cronicidade a incapacidade funcional pulmonar após 1 ano de alta hospitalar. A partir disso, fica claro o benefício da implementação da reabilitação pulmonar como meio de se minizar tais efeitos tão adversos [14,15].

\section{Referências}

1. Klok FA, Boon GJAM, Barco S, Endres M, Geelhoed JJM, Knauss S et al. The Post COVID-19 Functional Status (PCFS) Scale: a tool to measure functional status overtime after COVID-19. Eur Respir J 2020;56:2001494.

https://doi.org/10.1183/13993003.01494-2020

2. Simpson R, Robinson L. Rehabilitation following critical illness in people with COVID-19 infection. Am J Phys Med Rehabil 2020.

https://doi.org/10.1097/phm.0000000000001443

3. Kiekens C, Boldrini P, Andreoli A, Avesani R, Gamna F, Grandi M et al. Rehabilitation and respiratory management in the acute and early post-acute phase. "Instant Paper From the Field" on Rehabilitation Answers to the Covid-19 Emergency. Eur J Phys Rehabil Med 2020. https://doi.org/10.23736/S1973-9087.20.06305-4 
4. Barker-Davies RM, O'Sullivan O, Senaratne KPP, Baker P, Cranley M, Dharm-Datta S et al. The Stanford Hall consensus statement for post-COVID-19 rehabilitation. Br J Sports Med 2020;54:949-59. https://doi.org/10.1136/bjsports-2020-102596

5. Griffith JF. Musculo skeletal complications of severe acute respiratory syndrome. Semin Musculoskelet Radiol 2011;15:554-60. https://doi.org/10.1055/s-0031-1293500

6. Hill NS. Pulmonary rehabilitation. Proc Am Thorac Soc 2006;3:66-74. https://doi.org/10.1513/pats.200511-121JH

7. Yang LL, Yang T. Pulmonary rehabilitation for patients with coronavirus disease 2019 (COVID-19). Chronic Diseases and Translational Medicine 2020;6(2):79-86. https://doi.org/10.1016/i.cdtm.2020.05.002

8. Spruit MA, Singh SJ, Garvey C et al. An official American Thoracic Society/European Respiratory Society statement: key concepts and advances in pulmonary rehabilitation. Am J Respir Crit Care Med 2013;188:e13ee64

9. Sheehy LM. Considerations for post acute rehabilitation for survivors of COVID-19. JMIR Public Health Surveill 2020;6(2):e19462. https://doi.org/10.2196/19462

10. Chinese Association of Rehabilitation Medicine. Respiratory Rehabilitation Committee of Chinese Association of Rehabilitation Medicine. Cardiopulmonary Rehabilitation Group of Chinese Society of Physical Medicine Rehabilitation Recommendations for respiratory rehabilitation of COVID-19 in adult. 2020;3(43):e029. https://doi.org/10.3760/cma.j.cn112147-20200228-00206

11. Lazzeri M, Lanza A, Bellini R, Bellofiore A, Cecchetto S, Colombo A et al. Respiratory physiotherapy in patients with COVID-19 infection in acute setting: a position paper of the Italian Association of Respiratory Physiotherapists (ARIR). Monaldi Arch Chest Dis 2020;26;90(1):1285. https://doi.org/10.4081/monaldi.2020.1285

12. Zhao $\mathrm{H}$, Xie $\mathrm{Y}$, Wang $\mathrm{C}$. Recommendations for respiratory rehabilitation in adults with COVID-19. Chin Med J 2020. https://doi.org/10.1097/CM9.0000000000000848

13. Healthy China Initiative (2019-2030). The People's Republic of China: National Health Commission of the People's Republic of China 2019. http://www.nhc.gov.cn/guihuaxxs/s3585u/201907/e9275fb95d5b4295be8308415d4cd1 b2.shtml

14. Lee N, Hui D, Wu A et al. A major outbreak of severe acute respiratory syndrome in Hong Kong. N Engl J Med 2003;348:1986-994. https://doi.org/10.1056/nejmoa030685

15. Herridge MS, Cheung AM, Tansey CM et al. One-year outcomes in survivors of the acute respiratory distress syndrome. N Engl J Med 2003;348:683-93. https://doi.org/10.1056/nejmoa022450 